\title{
Jets in Deep Inelastic Scattering and High Energy Photoproduction at HERA *
}

\author{
Gerd W. Buschhorn \\ Max-Planck-Institut für Physik \\ (Werner-Heisenberg-Institut) \\ Föhringer Ring 6 \\ 80805 München
}

\begin{abstract}
Recent results on jet production in neutral current deep inelastic scattering and high energy photoproduction at the HERA electron-proton-collider are briefly reviewed. The results are compared to $\mathrm{QCD}$ expectations in $\mathrm{NLO}$ and $\alpha_{s}$ determinations using these data are summarized.
\end{abstract}

\section{Introduction}

The hard partonic interactions at the center of photon-induced hadronic processes in deep inelastic electron/positron-proton-scattering are charaterised by jets. The virtuality $Q^{2}$ of the exchanged photon can be varied from high values in deep inelastic scattering (DIS) to zero in photoproduction. According to the factorization theorem the hadronic process can be factorized into a pertubatively treated hard part, which includes the evolution of the parton entering the hard process, and a soft part, which describes the parton distribution of the target nucleon; the characteristic energy for this separation is given by the factorization scale. In DIS processes involving light quarks only, an energy scale is provided by $Q^{2}$ or the transverse energy of the emerging hadronic jet (or jet system), whereas in photoproduction the jet energy is the only available energy scale; in heavy quark production the quark mass may provide an additional energy scale. In jet studies details of the hard QCD calculations and their matching to the evolution of the target partons are probed; the investigations may also provide information on the parton distribution functions (pdfs) of the proton and photon and can serve to measure $\alpha_{s}$ and its scale dependence.

In this report, recent results from the H1- and ZEUS-collaboration on the production of standard jets i.e. jets without heavy quark tagging in neutral current DIS processes and photoproduction are discussed; jets from diffractive processes and jet shapes are not included.

${ }^{*}$ Talk given at the 9th Adriatic Meeting "Particle Physics and the Universe" in Dubrovnik/Croatia, 4.-24. September 2003 
colour coherence in the evolution ladder is taken into account imposing an angularordering of the gluon emission with the maximum angle determined by the hard scattering subprocess; the cross section factorizes into pdfs unintegrated both in $k_{\mathrm{T}}$ and the gluon angle and an off-shell matrix element. At asymptotic energies CCFM evolution approaches BFKL at small $x$ and DGLAP at large $x$ and $Q^{2}$.

While at high $Q^{2}$ the virtual photon behaves as a pointlike particle, at low $Q^{2}$ and in particular in photoproduction with $Q^{2}=0$, its hadronic structure has to be taken into account. The momentum transfer from the scattered electron to the parton cascade then takes place via a parton of the "resolved photon", which itself may evolve in a parton cascade towards the hard scattering process. Such resolved processes contribute a background to hard scattering processes but also can serve as a tool to investigate the photon structure function.

The response of the detectors to jet events is studied with Monte Carlo generated events, which are based on the measured pdfs of the proton and photon and on simulations of the fragmentation and hadronization of the partons. In RAPGAP the QCD matrix element is calculated in LO with NLO corrections evaluated by perturbative generation of parton showers; the hadronization of the partons is performed via JETSET, which is based on the Lund-string model. In ARIADNE, the parton cascade is generated using the colour-dipole-model (CDM) while in CASCADE it is calculated via CCFM evolution.

\section{Jets in DIS}

The inclusive jet cross section $\sigma_{j}$ results from the convolution of the measured pdfs of the proton $f_{a}\left(x_{a}, \mu_{F}\right)$ with the pertubatively calculated hard partonic cross section $d \sigma_{a}\left(x_{a}, \alpha_{s}, \mu_{R}, \mu_{F}\right)$,

$$
\sigma_{j}=\sum_{a} \int d x f_{a}\left(x, \mu_{F}^{2}\right) d \sigma_{a}\left(x, \alpha_{s}, \mu_{R}^{2}, \mu_{F}^{2}\right)\left(1+\delta_{\text {hadr }}\right)
$$

where the sum is to be taken over all partons $a$ of the proton; $\sigma_{j}$ has to be folded with the bremsstrahlungsspectrum. $\mu_{R}$ is the renormalization scale and $\mu_{F}$ the factorization scale. $\left(1+\delta_{\text {hadr }}\right)$ represents the nonperturbative correction accounting for the hadronization of the partons; it is estimated from MC generators.

(i) Single jet cross sections have been measured by $\mathrm{H1}$ and ZEUS in the Breit frame using the $k_{\mathrm{T}}$-cluster algorithm. The kinematical parameters for the $\mathrm{H} 1$ data [5] are $150<Q^{2}<5000 \mathrm{GeV}^{2}, 7<E_{T}<50 \mathrm{GeV},-1<\eta_{\text {lab }}<2.5$, while for the ZEUS data [6] they are $Q^{2}>125 \mathrm{GeV}^{2}, E_{T}>8 \mathrm{GeV},-2<\eta_{\text {lab }}<1.8$. The good agreement of both measurements with NLO QCD calculations (DISENT) at high $Q^{2}$ values (fig. 2) has enabled precision determinations of $\alpha_{s}$ and its scale dependence from these data (see Sect. 5).

The ZEUS data have been used to study the azimuthal distribution of jets in the Breit frame [7]. The measured $\phi$-distribution agrees well with the NLO QCD predictions from DISENT with either $\mu_{R}=E_{T}$ or $Q$, providing an independent test of QCD. 


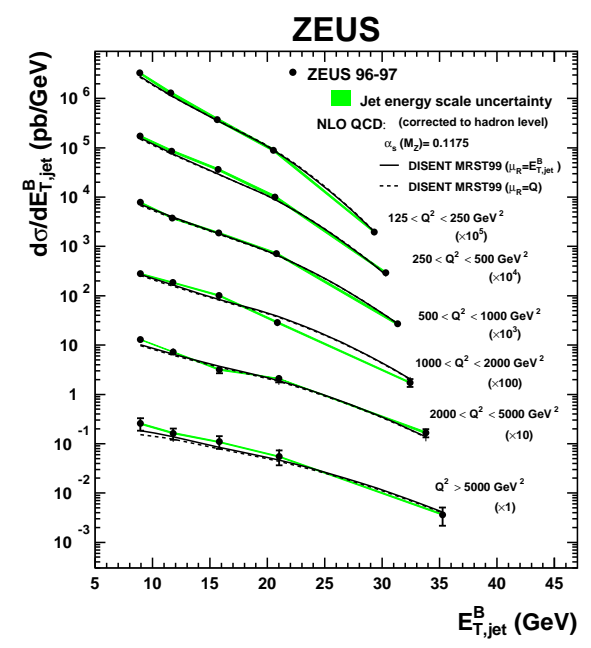

Figure 2: Inclusive jet cross section in DIS in the Breit system (fig. from [6])

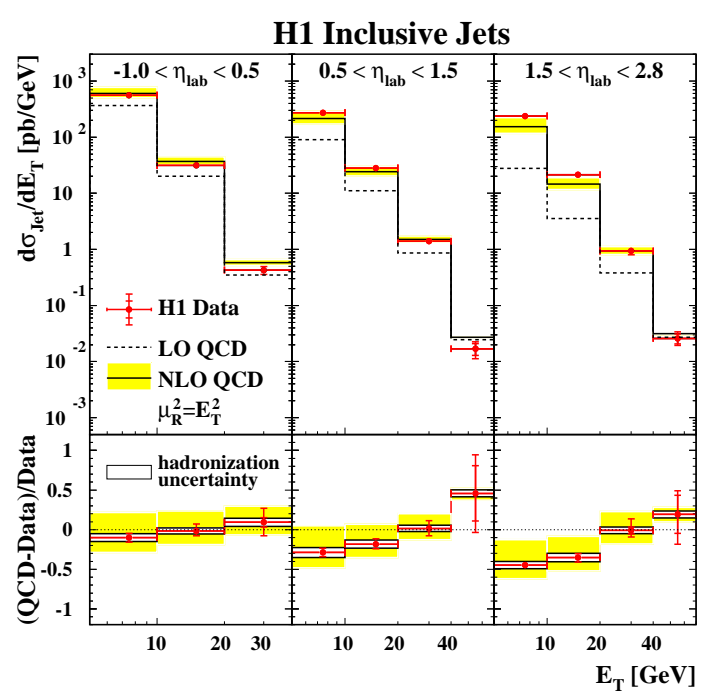

Figure 3: Inclusive jet cross section in DIS in the Breit system integrated over $5<Q^{2}<$ $100 \mathrm{GeV}^{2}$ and $0.2<\eta_{\text {lab }}<0.6$, NLO QCD: DISENT without hadronization correction; shaded band: change of renorm. scale by $1 / 2$ and 2 (fig. from [18) 


\section{ZEUS}

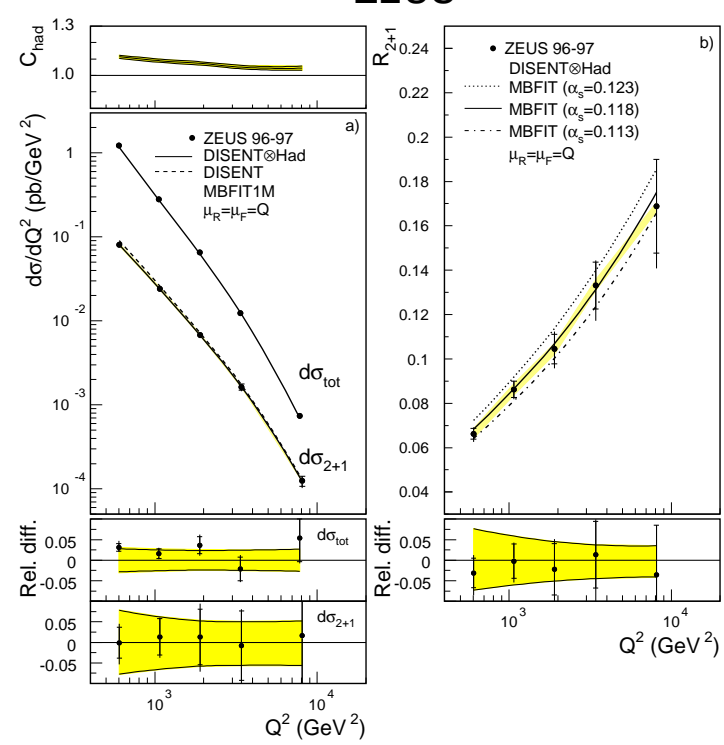

Figure 5: Inclusive jet $\left(d \sigma_{t o t}\right)$ and dijet $\left(d \sigma_{2+1}\right)$ cross sections with dijet fraction $R_{2+1}$ in DIS; shaded band: uncertainty in absolute energy scale of jets (fig. from [1])

Dijet data have been taken by H1 [1] and ZEUS [1] in similar kinematical regions and analyzed by similar methods. Asymmetric cuts have been applied in $E_{T}$ in order to avoid infrared sensitive regions of the phase space. At not too low $Q^{2}$ and $E_{T}$ i.e. $Q^{2}>10 \mathrm{GeV}^{2}$ and $E_{T}>5 \mathrm{GeV}$, the data are reproduced (fig. 5) within $10 \%$ by NLO QCD (DISENT). For the $\alpha_{s}$ analysis of these data see Sect. 5 ,

Recent dijet-data from H1 12 in the low- $x$ region i.e. $10^{-4}<x<10^{-2}$ for $10<Q^{2}<100 \mathrm{GeV}^{2}$ agree within errors with DGLAP based NLO QCD predictions for inclusive cross sections. Serious disagreement is observed, however, in the ratio $S$ of the number of dijet events with azimuthal separation $\phi<2 \pi / 3$ to the total number of dijet events. While the LO (DISENT) prediction is much to low, the agreement is improved except in the low $-x$ and low- $Q^{2}$ region if a third hard jet is included in the NLO (NLOJET) calculation (fig. 6). A comparison with RAPGAP shows that the inclusion of resolved processes improves the agreement except in the very low- $x$ and low- $Q^{2}$ region. From a comparison of the data with the CCFM based CASCADE MC a strong sensitivity to the assumptions on the unintegrated gluon distribution can be inferred, which may be used to constrain the gluon pdf. ARIADNE, which is based on CDM, describes the low- $x$ and $-Q^{2}$ region reasonably well, fails, however, at larger $Q^{2}$ values.

The jet studies in deep inelastic scattering have been extended to trijets [13, 14. as well as to subjets [15].

\section{Jets in photoproduction}

The description of photoproduction involves the convolution of the hard partonic scattering cross section with the pdfs of both the proton and the photon. In standard jet production i.e. jets from light quarks only, for the energy scale $\mu_{R}=\mu_{F}=\mu=$ 

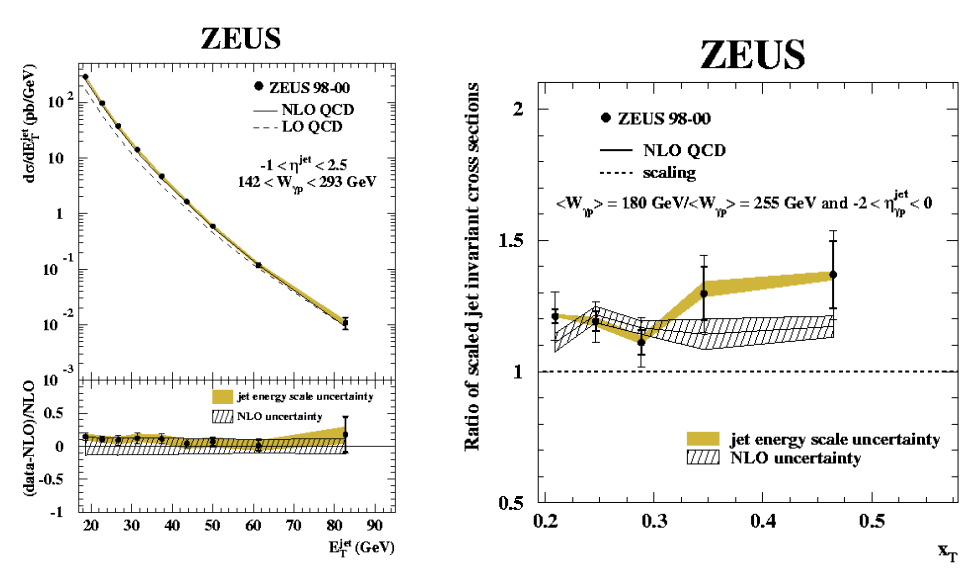

Figure 7: Inclusive jet production cross section in photoproduction. Ratio of scaled invariant cross sections (av. over $-2<\eta_{\text {lab }}<0$ ) for the two shown $\left\langle W_{\gamma p}\right\rangle$ compared with NLO QCD; (fig. from [16])

$x_{\mathrm{T}}=2 E_{T} / W_{\gamma p}$ at $\left\langle W_{\gamma p}\right\rangle=180$ and $255 \mathrm{GeV}$. The cross section ratio as function of $x_{T}$ violates scaling as predicted from QCD (fig. [7, right).

(ii) The dijet photoproduction cross section is dependent on the dijet angle $\theta^{*}$ in the parton-parton-cms, which is sensitive to the matrix element of the hard scattering subprocess. Direct photon processes are dominated by BGF with the angular dependence of the spin $1 / 2$-progator $\sim\left(1-\cos \theta^{*}\right)^{-1}$ whereas in resolved processes gluon-exchange with the steeper angular dependence of the spin 1-progator $\sim\left(1-\cos \theta^{*}\right)^{-2}$ is dominating. The dijet events can be enriched with direct or resolved processes by cutting on the fraction $x_{\gamma}^{\text {obs }}$ of the photon-momentum transferred to the two jets of highest transverse energy, which is given by $x_{\gamma}^{o b s}=$ $\left(E_{T 1} e^{-\eta_{1}}+E_{T 2} e^{-\eta_{2}}\right) / 2 y E_{e}$ with $y$ the fractional electron energy carried by the photon in the $p$-rest frame.

Dijet photoproduction has been studied by ZEUS [18] and H1 [19] under similar kinematical conditions using the $k_{\mathrm{T}}$-cluster algorithm. The differential cross sections in the dijet invariant mass, $E_{T}$ and $\eta$ agree well with NLO QCD expectations and in particular the measured dijet angular distributions (fig. 8) shows the expected difference for direct $\left(x_{\gamma}^{o b s}>.75\right)$ and resolved processes $\left(x_{\gamma}^{o b s}<.75\right)$. The sensitivity of the data to the parametrization of the photon-pdfs appears to be affected by the choice for the $E_{T}$-cuts (ZEUS: $E_{T 1}>14 \mathrm{GeV}, E_{T 2}>11 \mathrm{GeV} ; \mathrm{H} 1: E_{T 1}>25 \mathrm{GeV}$, $\left.E_{T 2}>15 \mathrm{GeV}\right)$.

\section{$4 \quad \alpha_{s}$-determinations}

The good agreement of jet cross sections with QCD predictions has enabled determinations of $\alpha_{s}$ and its scale dependence.

The H1 inclusive jet cross sections [5] as a function of $E_{T}$ have been fitted to the QCD prediction for four regions of $Q^{2}$ using the proton-pdfs and $\mu_{R}$ and $\mu_{F}$ as input. The resulting $\alpha_{s}$ has been shown to be stable against variations of the jet 


\section{ZEUS}

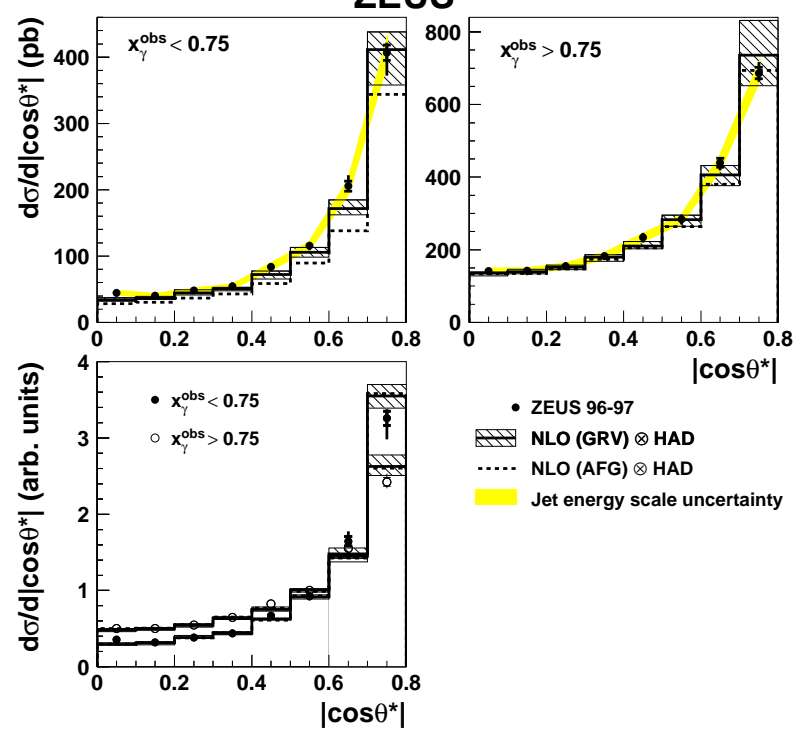

Figure 8: Inclusive dijet photoproduction as a function of the dijet angle $\theta^{*}$ in the partonparton-cms for different cuts on the fractional photon momentum $x_{\gamma}^{o b s}$ transferred to the dijet system. Comparison with NLO QCD for different photon-pdfs; (fig. from [18])

algorithm. The combined fit to all $Q^{2}$-data evolved to $m_{Z}$ is shown in the summary fig. 91).

For the analysis of the ZEUS data [6] the following procedure has been applied: The cross sections $d \sigma / d A$ with $A=Q^{2}, E_{T}$ have been calculated in NLO QCD for the same pdf-set with three $\alpha_{s}$-values. These calculations were used to parametrize the $\alpha_{s}$-dependence of the binned cross sections $(d \sigma / d A)_{i}$ for each bin $i$ according to $\left(d \sigma\left(\alpha_{s}\right) / d A\right)_{i}=C_{1 i} . \alpha_{s}^{1}+C_{2 i} . \alpha_{s}^{2}$ with $\alpha_{s}=\alpha_{s}\left(M_{Z}\right)$. From a $\chi^{2}$-fit of this ansatz to the measured $d \sigma / d A, \alpha_{s}$ was obtained for the chosen regions of $A$. The best fit was obtained for $Q^{2}>500 \mathrm{GeV}^{2}$ (fig. 9). The same procedure has been applied to determine the scale dependence of $\alpha_{s}$. With $E_{T}$ as energy scale, the $\alpha_{s}$ dependence of $d \sigma / d E_{T}$ was parametrized in terms of $\alpha_{s}\left(\left\langle E_{T}\right\rangle\right)$ where $\left\langle E_{T}\right\rangle$ is the mean value of $E_{T}$ in bin $i$. The result is shown in fig. 10.

The same method has been applied to other jet results from ZEUS i.e. the dijet fraction [12, subjet multiplicities ([15]) and jets from photoproduction [15]. A summary of the results is shown in figs. 9 and 10

\section{Summary}

In the analysis of jet production in DIS and photoproduction at HERA the longitudinally invariant $k_{\mathrm{T}}$-cluster algorithm in its inclusive version has become the standard jet finding algorithm.

In DIS, the inclusive jet cross sections at higher values of $Q^{2}$ and $E_{T}$ are well described by NLO QCD; in this region higher order and hadronization corrections are small; this holds as well for photoproduction. 


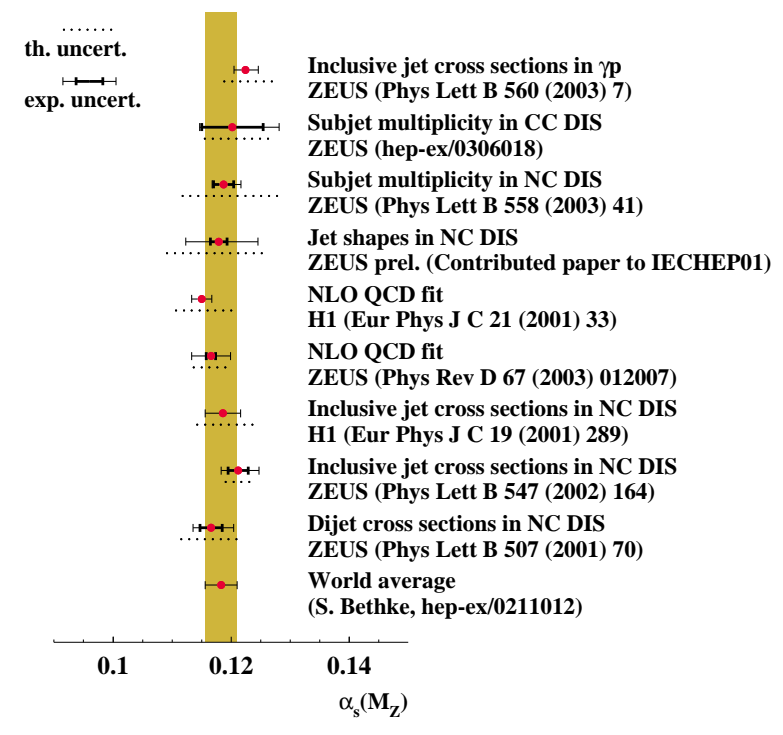

Figure 9: $\alpha_{s}\left(M_{Z}\right)$ from recent H1 and ZEUS measurements and the world average

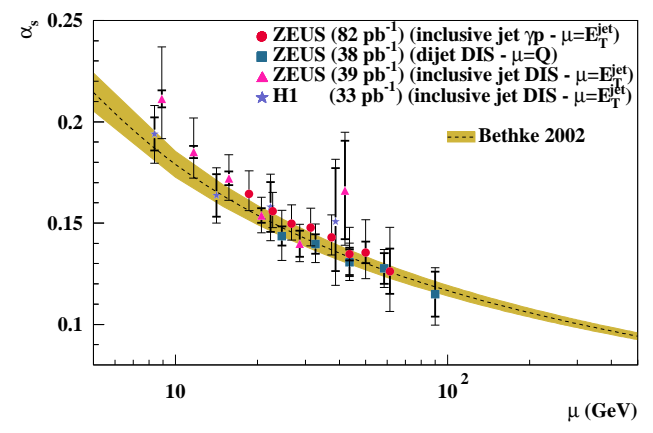

Figure 10: Scale dependence of $\alpha_{s}$ from recent H1 and ZEUS measurements and QCD predictions for $\alpha_{s}\left(M_{Z}\right)=0.1183$ (world average) 
In the forward region and/or at smaller $Q^{2}$ resp. $x$ values, the situation is less satisfactory. DGLAP based calculations are expected to become less reliable in this region, the mentioned corrections are sizable and the hadronic structure of the photon i.e. resolved processes have to be taken into account. Calculations based on BFKL or CCFM evolution only partly show better agreement. An increase in experimental statistics and efforts to reduce the theoretical uncertainties are highly desirable for a better understanding of this challenging region.

The measurements of $\alpha_{s}$ and its running from jet data have yielded results which are of perhaps unexpected precision; they can well compete with other precision measurements e.g. from $e^{+} e^{-}$-annihilation and are in good agreement with the world average.

Acknowledgements I thank G. Grindhammer for valuable discussions and comments on the paper.

\section{References}

[1] S.D. Ellis and D.E. Soper, Phys. Rev. D48, 3160 (1993) hep-ph/9305266;

S. Catani et al., Nucl. Phys. B406, 187 (1993)

[2] V. Gribov and L.N. Lipatov, Sov. J. Nucl. Phys. 15, 438, 675 (1972);

L.N. Lipatov, Sov. J. Nucl. Phys. 20, 94 (1975);

G. Altarelli and G. Parisi, Nucl. Phys. B126, 298 (1977);

Y.L. Dokshitzer, Sov. Phys. JETP 46, 641 (1977)

[3] V.S. Fadin, E.A. Kuraev and L.N. Lipatov, Sov. Phys. JETP 44, 443(1976);

V.S. Fadin, E.A. Kuraev and L.N. Lipatov, Sov Phys. JETP 45, 199 (1977);

Y. Balitsky and L.N. Lipatov, Sov. J. Nucl. Phys. 28, 822 (1978)

[4] M. Ciafolini, Nucl. Phys. B296, 49 (1988);

S. Catani, F. Fiorani and G. Marchesini, Phys. Lett. B234, 339 (1990);

S. Catani, F. Fiorani and G. Marchesini, Nucl. Phys. B336, 18 (1990);

G. Marchesini, Nucl. Phys. B445, 49 (1995) hep-ph/9412327

[5] C. Adloff et al., Europ. Phys. J. C19, 289 (2001) [DESY 00-145; hep-ex/0010054]

[6] S. Chekanov et al., Physics Letters B547, 164 (2002) [DESY 02-112; hep-ex/0208037]

[7] S. Chekanov et al., Physics Letters B551, 226 (2003) [DESY 02-171; hep-ex/0210064]

[8] C. Adloff et al., Physics Letters B542, 193 (2002), [DESY 02-079; hep-ex/0206029]

[9] J. Jung: H1 Collaboration, ICHEP 02, Amsterdam (2002)

[10] L. Goerlich: H1 Collaboration, DIS 2003, St. Petersburg (2003)

[11] J. Breitweg et al., Physics Letters B507, 70 (2001) [DESY 01-018; hep-ex/0102042];

S. Chekanov et al., Europ. Phs. J. C23, 13 (2002) [DESY 01-127; hep-ex/0109029] 
[12] A. Aktas et al.: H1-collaboration DESY 03-160; hep-ex/0310019

[13] C. Adloff et al., Physics Letters B515, 17 (2001) [DESY 01-073; hep-ex/0106078

[14] N. Krumnack: ZEUS Collaboration, DIS 2003, St. Petersburg (2003)

[15] S. Chekanov et al., Physics Letters B558, 41 (2003) [DESY 02-217; hep-ex/0212030]

[16] S. Chekanov et al., Physics Letters B560, 7 (2003) [DESY 02-228; hep-ex/0212064]

[17] C. Adloff al., submitted to Europ. Phys. J. C [DESY 02-225; hep-ex/0302034]

[18] S. Chekanov et al., Europ. Phys. J. C23, 615 (2002) [DESY 01-220; hep-ex/0112029]

[19] C. Adloff et al., Europ. Phys. J. C25, 13 (2002) [DESY 01-225; hep-ex/0201006 